\title{
Configurational affects on the compaction response of $\mathrm{CeO}_{2}$ powders
}

\author{
D.A. Fredenburga ${ }^{\mathrm{a}}$, D. Dennis-Koller, and D. Dattelbaum \\ Los Alamos National Laboratory: Shock and Detonation Physics, NM 87545, USA
}

\begin{abstract}
Initial configuration, which can include particle size and shape, initial density, and void location, can affect the measured compaction responses of initially porous materials. In this work, both the low- and high-strain-rate compaction response of several different morphology $\mathrm{CeO}_{2}$ powders are investigated experimentally. Quasi-static compaction curves are found to exhibit distinct differences between the morphologies, where initial packing efficiencies and particle aspect ratios are found to dominate the low pressure response. At low-strain-rates, the largest particles with the highest aspect ratio are found to exhibit the stiffest response, while those that most resemble spherical particles offer the least resistance to initial densification. At highstrain-rates a transition in compliance is observed, where smaller equiaxed particles are found to exhibit greater resistances to densification. The role of particle morphology and its affect on the communication of particle-level stresses during quasi-static and dynamic densification are discussed, and emphasis is placed on the mechanisms that cause the morphology-based transition in compliance.
\end{abstract}

\section{Introduction}

The consolidation of powders at intermediate pressures, comparable to the yield properties of the bulk material, is complicated by numerous factors inclusive of the strain rate under which consolidation occurs and the particle size, shape, and strength. At low-strain-rates it is believed that compaction occurs in three stages [1], where the time scales under which pressures are applied yields a quasihomogeneous distribution of pressure throughout the compact. This is in stark contrast to the loading of powders under high-strain-rates, where compaction occurs in a single step as the shock wave transforms the material from its initial to final state in several nanoseconds. Therefore, development of physically based models to describe the compaction of powdered materials at both low- and highstrain-rates requires detailed experimental studies over a wide range of strain rates on materials that have been wellcharacterized. This work offers such an investigation on three distinct morphology $\mathrm{CeO}_{2}$ powders.

\section{Materials characterization}

Three separate commercially available $\mathrm{CeO}_{2}$ powders were studied in the current investigation, each with their own unique morphology. The powders were chosen to reflect a wide range in initial morphology and particle size distributions, and will henceforth be referred to as $300 \mathrm{~nm}$, equiaxed, and rods. The $300 \mathrm{~nm}$ powder was obtained from Ocean State Abrasives (West Warwick, RI) and was listed with a purity of $99.95 \%$. The equiaxed powder was purchased from American Elements (Los Angeles, CA) with a purity of $99.5 \%$. The rods were obtained from Alfa Aesar (Ward Hill, MA) and was listed at $99.99 \%$ purity. Micrographs of the three powders in their as-received configuration are shown in Fig. 1. From Fig. 1 it is observed that the powders vary significantly is their size, shape, and aspect ratio. In comparing Figs. 1(a) and (b), one can see

a e-mail: dafreden@lanl.gov
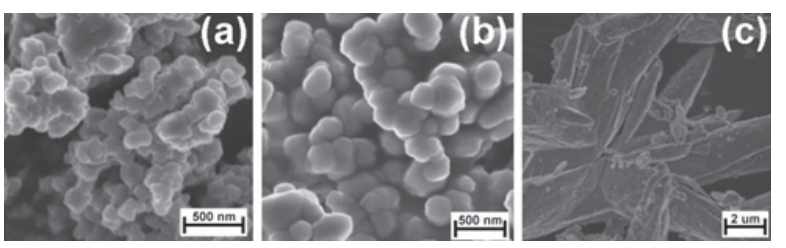

Fig. 1. As-received powders showing size and morphology of the (a) $300 \mathrm{~nm}$ powder, (b) equiaxed powder, and (c) rods; scale bars are $500 \mathrm{~nm}, 500 \mathrm{~nm}$, and $2 \mu \mathrm{m}$, respectively.

that the $300 \mathrm{~nm}$ powders are more irregular in shape than the equiaxed powders, and are characterized by a globular appearance. Visual inspection of the two powders reveals both the $300 \mathrm{~nm}$ and equiaxed powders are of comparable size; however, the $300 \mathrm{~nm}$ particles appear to have a greater percentage of smaller particles. Particle size for the rods is the largest of the powders investigated, and its morphology is characterized by high aspect ratios in the longitudinal direction, Fig. 1(c).

To gain further insight into the initial particle configuration, a series of particle size analysis experiments were performed. The analysis was conducted using a light scattering technique with a Horiba Light Scattering Analyzer. Tests were performed on the as-received powders first, following submersion in a solution of isopropanol alcohol, and subsequently after varying degrees of sonication. For all materials it was found that some degree of agglomeration was present in the as-received powders, and both the mean and mode particle sizes were found to decrease with increasing sonication time. Typical sonication time to reach an equilibrium particle distribution was between 2-3 minutes for the $300 \mathrm{~nm}$ and equiaxed particles, while the rods required times as long as 10 minutes. Results of the equilibrated particle size distributions for the three powders are shown in Fig. 2. Note that in using a light scattering technique reported particle sizes are based on an assumed spherical geometry.

Examination of the particle size analysis shown in Fig. 2 reveals that both the $300 \mathrm{~nm}$ and equiaxed powder are bi-modal in character, while the rods are nominally 

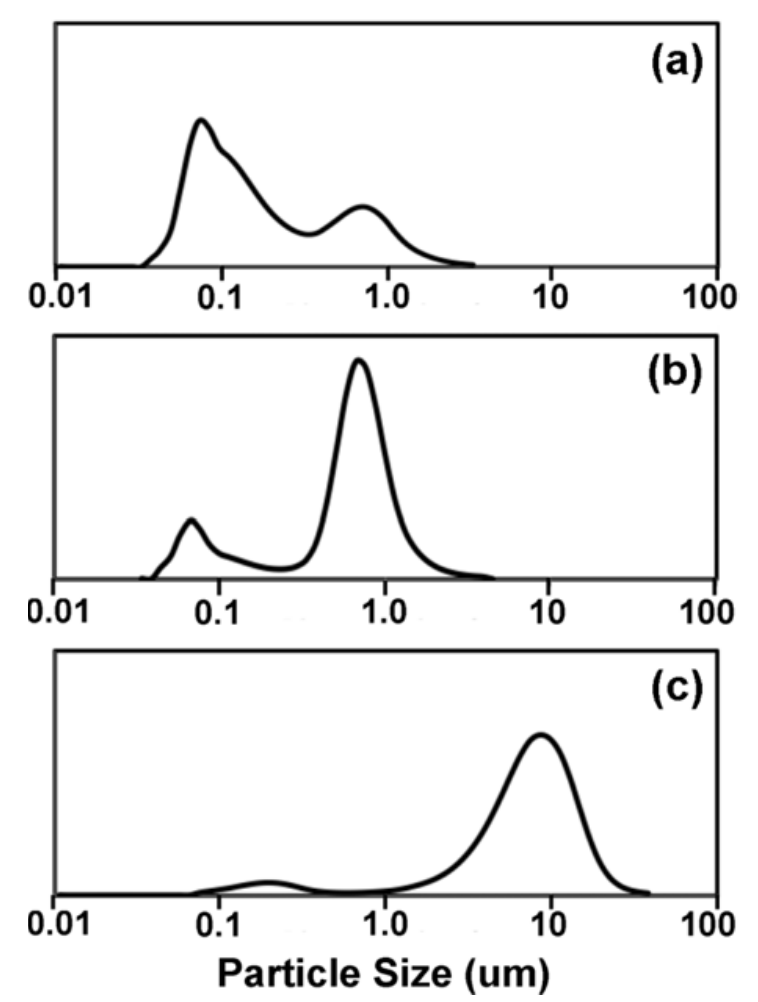

Fig. 2. Particle size distribution following sonication and equilibration for (a) $300 \mathrm{~nm}$ powder, (b) equiaxed powder, and (c) rods. Relative intensity of peaks (y-axis) is the same for all powders.

uni-modal. Interestingly, modes for the $300 \mathrm{~nm}$ and equiaxed powders are similar, where the $300 \mathrm{~nm}$ powder has modes at 0.08 and $0.70 \mu \mathrm{m}$, and the modes for the equiaxed powder are 0.07 and $0.70 \mu \mathrm{m}$. While the modes for these two powders are nearly identical, the relative intensities of each mode differ. The $300 \mathrm{~nm}$ powder has the greatest intensity at smaller particle sizes, while the situation is reversed for the equiaxed particles. This behavior is exemplified further by the mean particle size, which for the $300 \mathrm{~nm}$ and equiaxed particles are 0.32 and $0.63 \mu \mathrm{m}$, respectively. For the rods, Fig. 2(c), the primary mode is found at $8.24 \mu \mathrm{m}$, and a slight tail is observed at around $0.03 \mu \mathrm{m}$. While the mean particle size for the rods reported by light scattering was $7.8 \mu \mathrm{m}$, examination of Fig. 1(c) reveals a morphology for the rods that is composed of high aspect ratio particles. Thus, the mean and mode particle sized found by light scattering, which approximate the particle as a sphere, do not accurately reflect the actual dimensions of the rods.

The measured differences in particle size, size distribution, and shape are also accompanied by variations in specific surface area and resting particle configuration. Table 1 shows the specific surface area and bulk and tap densities for the three morphology powders. Surface area was measured using a gas adsorption technique with a Quantachrome Autosorb-1 MP analyzer, which reflects the surfaces of the many facets and open porosity for a given particel. For the particles investigated, surface areas range from $3.34 \mathrm{~m}^{2} / \mathrm{g}$ for the equiaxed particles to $10.7 \mathrm{~m}^{2} / \mathrm{g}$ for the $300 \mathrm{~nm}$ particles.

In interpreting the surface area measurements it is important to recall that surface areas tends to increase
Table 1. Table of measured specific surface area (S.A.) and bulk and tap density for the three morphology powders.

\begin{tabular}{lccc}
\hline Powder & $\begin{array}{c}\text { S.A. } \\
\left(\mathrm{m}^{2} / \mathrm{g}\right)\end{array}$ & $\begin{array}{c}\text { Bulk Density } \\
\left(\mathrm{g} / \mathrm{cm}^{3}\right)\end{array}$ & $\begin{array}{c}\text { Tap Density } \\
\left(\mathrm{g} / \mathrm{cm}^{3}\right)\end{array}$ \\
\hline $300 \mathrm{~nm}$ & 10.7 & 1.00 & 1.41 \\
Equiaxed & 3.34 & 1.27 & 2.13 \\
Rods & 6.28 & 1.01 & 1.62 \\
\hline
\end{tabular}

as particle size is reduced and as particle geometry deviates further from spherical. Therefore, it is not surprising that the equiaxed particles are found to have the lowest measured values as they possess a moderate particle size and their geometry most closely resembles that of a sphere. The highest surface areas are measured for the $300 \mathrm{~nm}$ powders. Their small particle size, centered around $0.08 \mu \mathrm{m}$, coupled with an irregular geometry (Fig. 1(a)), results in a relatively high amount of surface area per unit volume. Surface area measurements for the rods reveal an intermediate value as their relatively large size tends to drive the surface area down, while the high aspect ratio produces an opposing effect.

Bulk and tap densities for the powders were measured using a Quantachrome Autotap 02106-60-1 equipped with a $10 \mathrm{ml}$ graduated cylinder for performing measurements on limited amounts of powders. Bulk densities were calculated by pouring a known mass of powder into the graduated cylinder and measuring the respective volume. Subsequently, a series of 3,000 taps were performed on the bulk powder, and a measurement of the tap density was recorded. In addition to providing information about the resting configuration of the particles, measurements of bulk and tap densities, specifically the change in density from bulk to tap, yields insight into the compaction behavior of the particles.

For low-strain-rate compaction, consolidation occurs in three stages [1], where the first stage is characterized by particle rearrangement. Thus, examining the relative amount of compaction that occurs as a result of rearrangement during the tapping process should give some measurement of the relative ease with which the initial stage of compaction occurs. From the densities reported in Table 1, the relative increases in density as a result of tapping for the $300 \mathrm{~nm}$, equiaxed, and rods are are found to be $41 \%$, $68 \%$, and $60 \%$, respectively. Considering only the greatest and least density increases, examination of the particle size distributions can help explain why greater packing efficiencies are found for the equiaxed particles. It was noted earlier that both the equiaxed and $300 \mathrm{~nm}$ powders shared similar modes at approximately 0.08 and $0.7 \mu \mathrm{m}$. However, the bulk of the $300 \mathrm{~nm}$ powder was centered around the smaller particle size, while the equiaxed powder was centered around the larger size. Considering this size distribution, it appears that for these systems of $\mathrm{CeO}_{2}$ particles higher packing efficiencies are reached with a greater concentration of large particles accompanied by a reduced fraction of small particles. Indeed, this is exactly the result found by Santiso and Müller [2], who studied packing efficiencies in bi-modal systems of idealized spherical particles. 


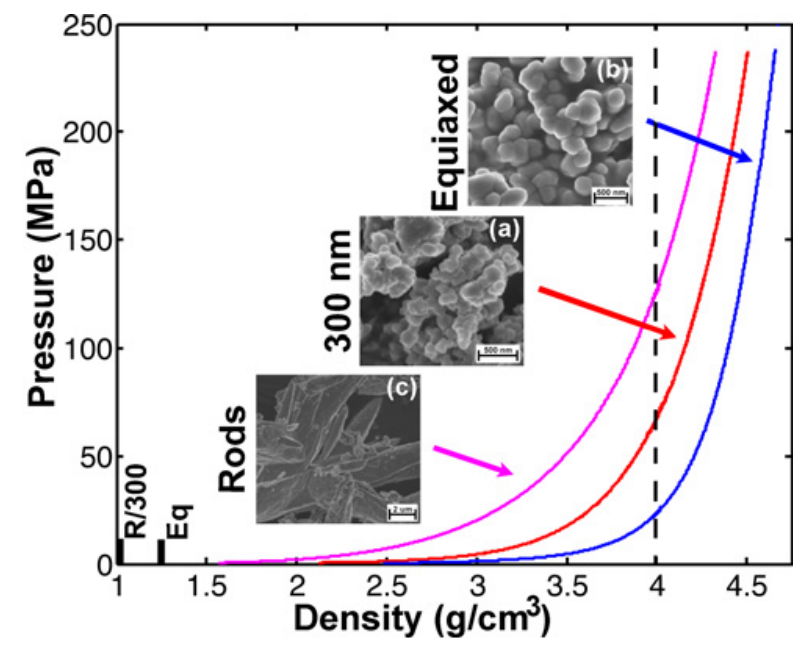

Fig. 3. Quasi-static compaction curves for the three morphology $\mathrm{CeO}_{2}$ powders. Bulk density values are shown in the bottom left for the $300 \mathrm{~nm}$ (300), equiaxed (Eq), and rods (R). Also shown are the pressures required to reach $4.0 \mathrm{~g} / \mathrm{cm}^{3}$, the initial density for dynamic experiments.

\section{Low-strain-rate compaction}

Due to the relatively long time scales and homogeneous stress distribution under which low-strain-rate compaction occurs, it is found that particle morphology strongly influences the path of densification in this regime. Figure 3 shows the consolidation response for the three powders up to $\sim 250 \mathrm{MPa}$. In these tests, approximately 5 grams of powder was inserted into a 1 " inner diameter punch and die set composed of high strength tool steel. The powder and die fixture were then loaded in a screwdriven Instron 4483 load frame with $150 \mathrm{kN}$ load capacity. Continuous measurements of the force and displacement were recorded for both the unfilled and powder filled die fixtures. Pressure-density curves for each powder were then calculated from the force-displacement data after accounting for compliance in the machine and empty die set. It was assumed that the die walls remained rigid during compression.

At the upper range of pressures shown in Fig. 3 slopes and curvatures for the three powders appear similar, suggesting similar mechanisms are dominating the densification response at higher pressures. However, in the low pressure regime significant deviations are observed between the three powders. Immediately apparent is that the densities at which the pressures begin to increase from non-negligible values are markedly different for each of the powders. The rods are observed to offer the greatest resistance to compaction, while the equiaxed particles offer the least. In comparing the rods with the $300 \mathrm{~nm}$ powder, which both start off at nearly the same bulk density, the rods begin to show a resistance to densification at lower densities than the $300 \mathrm{~nm}$ powder. This results in a densification path for the rods that is shifted to the left with respect to the $300 \mathrm{~nm}$ powder. For the $300 \mathrm{~nm}$ and equiaxed powders, the relative increase in density prior to an observable resistance to densification in similar. However, due to the greater initial packing efficiency in the equiaxed particles (higher bulk density), the densification path for the equiaxed particles is shifted to the right of the $300 \mathrm{~nm}$ curve.

Considering shape and curvature of the densification curves for the low, intermediate, and high pressures shown in Fig. 3, it is observed that the greatest differences between the curves occur at low pressures. Thus, it appears variation in the low-strain-rate compaction response can be largely attributed to the morphology characteristics that promote optimal packing efficiencies and particle rearrangement. Table 1 shows that the equiaxed particles exhibit both the greatest initial packing efficiencies (highest bulk density) as well as the highest propensity for rearrangement following tapping (greatest increase from bulk to tap density). Consequently, under quasistatic compaction its densification path lies furthest to the right, and it is able to reach the highest maximum density. However, using a similar metric to predict the low-strainrate compaction response for the $300 \mathrm{~nm}$ powder and rods would result in a densification path for the rods that lies to the right of the $300 \mathrm{~nm}$ powder due to the rods undergoing a greater amount of particle rearrangement during the tapping process. Therefore, additional considerations must be taken into account to explain the variation in lowstrain-rate compaction between the $300 \mathrm{~nm}$ particles and rods.

Rearrangement is the process of particles sliding past one another to fill void space. During the tapping operation this process occurs under the force of gravity, while during quasi-static compaction significantly higher forces are applied to the particles through the displacement of a punch. For particles $<100 \mu \mathrm{m}$ gravity is no longer the dominant force controlling particle movement, rather, Van der Waals forces $(<100 \mu \mathrm{m})$ and electrostatic forces $(<$ $1 \mu \mathrm{m}$ ) become the dominant forces, both of which can result in agglomeration [3]. Thus in the absence of applied pressures, rearrangement during the tapping operation for particles $<100 \mu \mathrm{m}$ is strongly influenced by both Van der Waals and electrostatic forces. Due to differences in particle size fractions, rearrangement during tapping for the $300 \mathrm{~nm}$ powder is likely influenced by both Van der Waals and electrostatic forces, where rearrangement for the rods may only be influenced by the former. However, during quasi-static compaction rearrangement occurs under the application of forces much greater than both Van der Waals and electrostatic, and the sliding of particles is controlled more by factors such as surface roughness and particle aspect ratio. Examination of Figs. 1(a) and (c) reveals that the rods possess much higher aspect ratios than the $300 \mathrm{~nm}$ powders and may also have an increased amount of surface roughness. Thus, under quasi-static compaction these factors cause the rods to undergo less rearrangement during the initial stages of compaction, and consequently result in the least compliant densification path.

\section{High-strain-rate compaction}

Dynamic experiments were performed using the $51 \mathrm{~mm}$ bore diameter gas-gun at Los Alamos National Laboratory [4]. Powders were loaded into the target fixture, as shown 


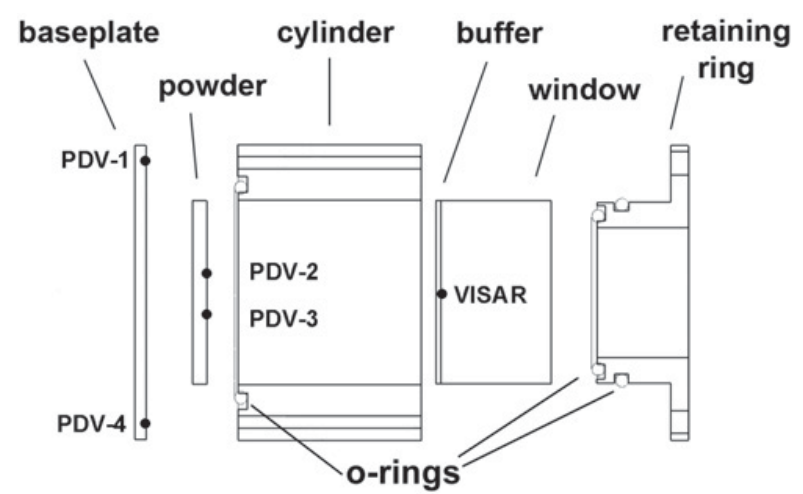

Fig. 4. Target fixture used for dynamic experiments shown in the expanded configuration, where location of surfaces probed by velocimetry are indicated with a solid dot and are labeled with corresponding velocimetry type.

in Fig. 4, to at an initial density of $\sim 4.0 \mathrm{~g} / \mathrm{cm}^{3}$, or $\sim 55 \%$ of theoretical maximum density (\% TMD). In these experiments, a $\mathrm{Cu}$ impactor was accelerated down the barrel of the gun and impacted the target fixture in Fig. 4 from left to right. In all instances an $\mathrm{OFHC} \mathrm{Cu}$ impactor struck an OFHC $\mathrm{Cu}$ baseplate, where the shock in the baseplate was transferred directly to the powder in the cell. Diameter of the pressed powder compact was $25.4 \mathrm{~mm}$, and nominal thicknesses for the baseplate, buffer, window, and powder were $1.5,0.5,16$, and $1.5-2.0 \mathrm{~mm}$.

Shock velocity in the powder was calculated from measurements of the arrival time of the $50 \%$ peak material velocity at the rear surface of the baseplate (PDV-1, PDV-4) and at the rear surface of the powder (PDV-2, PDV-3) using Photon Doppler Velocimetry (PDV) [5]. For the later experiments, two separate measurements of shock velocity were recorded from probe locations PDV-2 and PDV-3. However, in the initial experiments $(56-11-19,20$, 26,27 ) only a single measurement of the shock velocity was recorded. From measurements of the velocity of the impacting projectile and shock velocity in the powder, the impedance matching method was used to calculated the shock compressed state in the powder [6]. Uncertainties in the shock velocity for experiments where multiple shock velocity measurementsy were taken were determined using two standard deviations from the reported average value. For the experiments where only a single measurement was taken deviations from the measured $(50 \%)$ shock velocity at the $10 \%$ and $90 \%$ arrival times were used. These uncertainties, coupled with those in other measured quantities (impact velocity, initial density, powder thickness) and uncertainties in the calculated impactor and baseplate Hugoniots were used to determine the total uncertainty in the shock compressed powder in a manner consistent with the impedance matching method of Mitchell and Nellis [6].

In addition to the PDV probes which measured velocity histories at the baseplate rear surface and the powder rear surface, a single VISAR [7] probe was also employed, to investigate the wave structure after the shock had traversed through the powder and entered into the PMMA buffer and window. To accomplish this, a thin PMMA buffer $(0.5 \mathrm{~mm})$ was glued to the front surface of the PMMA window, and a reflective coating of $\mathrm{Al}$ was evaporated onto
Table 2. Tabular results for dynamic compaction experiments performed on all powders indicating measured initial density $\rho_{00}$, impact velocity $V_{I}$, and shock velocity $U_{S}$, and calculated values for the pressure $P$, and relative volume $V / V_{0}$ with respect to a solid density of $7.215 \mathrm{~g} / \mathrm{cm}^{3}$.

\begin{tabular}{lccccc}
\hline Shot & $\begin{array}{c}\rho_{00} \\
\left(\mathrm{~g} / \mathrm{cm}^{3}\right)\end{array}$ & $\begin{array}{c}\mathrm{V}_{I} \\
(\mathrm{~km} / \mathrm{s})\end{array}$ & $\begin{array}{c}\mathrm{U}_{S} \\
(\mathrm{~km} / \mathrm{s})\end{array}$ & $\begin{array}{c}\mathrm{P} \\
(\mathrm{GPa})\end{array}$ & $\mathrm{V} / \mathrm{V}_{0}$ \\
\hline $300 \mathrm{~nm}$ & & & & & \\
$56-11-20$ & 4.04 & 0.219 & 1.01 & 0.802 & 1.44 \\
$56-11-19$ & 4.02 & 0.407 & 1.27 & 1.82 & 1.29 \\
$56-11-56$ & 4.03 & 0.689 & 1.67 & 3.92 & 1.16 \\
Equiaxed & & & & & \\
$56-12-01$ & 4.03 & 0.394 & 1.24 & 1.73 & 1.29 \\
$56-12-06$ & 4.04 & 0.768 & 1.66 & 4.37 & 1.09 \\
Rods & & & & & \\
$56-11-26$ & 4.02 & 0.146 & 0.971 & 0.514 & 1.55 \\
$56-11-27$ & 4.05 & 0.405 & 1.23 & 1.78 & 1.27 \\
$56-11-55$ & 4.02 & 0.669 & 1.60 & 3.67 & 1.16 \\
\hline
\end{tabular}

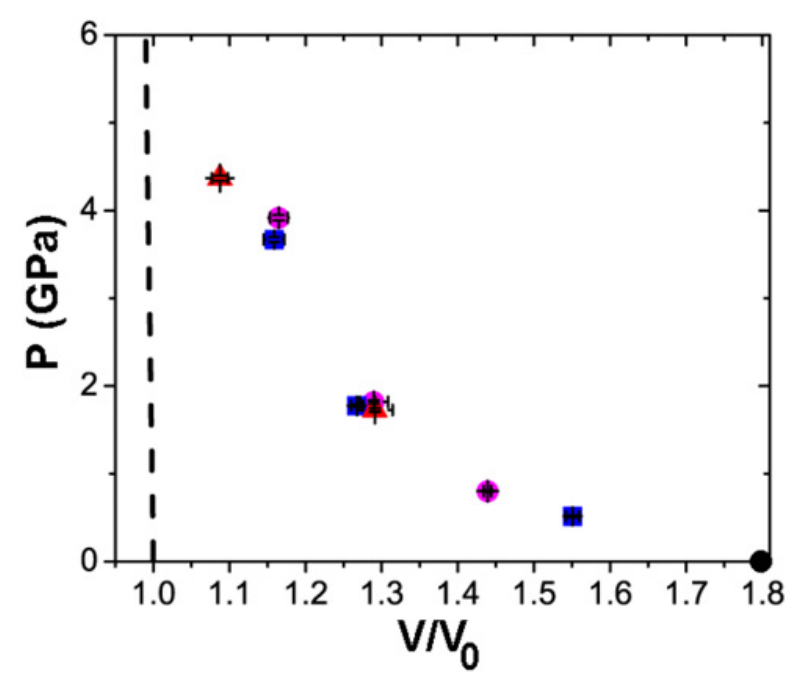

Fig. 5. Pressure-relative volume compaction response for the three morphology powders and calculated porous Hugoniot [8] (dashed line) for an initial density $\rho_{00}=4.0 \mathrm{~g} / \mathrm{cm}^{3}\left(\mathrm{~V} / \mathrm{V}_{0}=1.8\right)$ $\mathrm{CeO}_{2}$ powder The $300 \mathrm{~nm}$, equiaxed, and rods are represented by circles, squares, and triangles, respectively.

the window prior to affixing the buffer. This configuration allowed for relatively clean profiles of the transmitted wave to be measured.

Results from the dynamic experiments are given in Table 2, and are plotted in Fig. 5 with error bars reflecting the uncertainties previously discussed. The data reported in Table 2 retracts that from an earlier proceedings [8] as significant improvements in data analysis techniques have been made since the previous publication. From Fig. 5 it appears that minimal variation exists in the shock compaction response for the different morphology powders. At pressures below $2 \mathrm{GPa}$ the three powders seem to follow a nearly identical path of densification, where at approximately $1.8 \mathrm{GPa}$ the calculated compaction response for all three of the morphologies lie on top of one 
another, within the experimental uncertainties. As pressure is increased, the $300 \mathrm{~nm}$ and rods are observed to exhibit a slight difference in compaction response. For the $300 \mathrm{~nm}$ powder, a pressure of $3.92 \mathrm{GPa}$ is required to reach a relative volume of 1.16, while a similar compression for the rods is reached at $3.67 \mathrm{GPa}$. This suggests that as the amplitude of shock loading is increased in the compaction regime, one or more morphological aspects results in the rods being slightly more compliant than the $300 \mathrm{~nm}$ powder. The highest pressure data reported is for the equiaxed powder, where at $4.37 \mathrm{GPa}$ the material compresses to a relative volume of 1.09. At present, it is difficult to draw firm conclusions regarding the higher pressure compaction response for the three morphology powders due to a lack of overlap in $P-V / V_{0}$ space. Further experiments are planned for all three powders in this pressure regime, and additional results will be reported at the conference.

A closer look is now given to the two morphologies for which a measurable difference in dynamic compaction response was observed at higher pressures, the $300 \mathrm{~nm}$ (56-11-56) and rods (56-11-55). Referring to Table 1 in Sec. 2, one can see that the initial configurations for the as-received powders result in bulk densities that are nearly identical for the two morphologies. Under quasistatic loading the $300 \mathrm{~nm}$ powder is able to densify more efficiently at lower pressures, and consequently, is more easily compressed than the rods. Examination of the quasistatic densification curves shown in Fig. 3 reveal that in reaching an initial density of $4.0 \mathrm{~g} / \mathrm{cm}^{3}$ the rods require an increased pressure by nearly a factor of two, $130 \mathrm{MPa}$ (rods) vs. $70 \mathrm{MPa}(300 \mathrm{~nm})$. While the difference is more subtle under dynamic loading, the lower pressure quasistatic response contrasts sharply with the higher pressure dynamic response where under dynamic loading the rods are more easily compressed, $3.67 \mathrm{GPa}$ (rods) vs. $3.92 \mathrm{GPa}$ $(300 \mathrm{~nm})$ at $\mathrm{V} / \mathrm{V}_{0}=1.16$.

For quasi-static compaction it was found that the process of particle rearrangement had a strong influence on the final densities achievable, which was in turn affected by some mix of surface area, size distribution, and aspect ratio of the powders. In pre-pressing the powers for the dynamic experiments to an initial density of $4.0 \mathrm{~g} / \mathrm{cm}^{3}$ rearrangement of the particles has already taken place, and the configuration is in a semi-close packed arrangement. Therefore, under the rapid application of dynamic loads it is the network of particle interconnects that is expected to influence the high-strain-rate compaction response for the different morphology powders, where the structure of the network reflects the structure of the pressed powders. Thus, one must not only consider the initial morphology of the powders, but also the morphology of the precompressed $\left(4.0 \mathrm{~g} / \mathrm{cm}^{3}\right)$ powders in relating the measured compaction response to configurational aspects of the powders.

To determine the initial configuration of the powders prior to dynamic experiments, the powders were quasistatically loaded to $4.0 \mathrm{~g} / \mathrm{cm}^{3}$ and recovered for examination in an SEM. Micrographs for the pressed $300 \mathrm{~nm}$ and equiaxed powders revealed morphologies that were similar in appearance to the as-received powders. However, pressing the rods to $4.0 \mathrm{~g} / \mathrm{cm}^{3}$ resulted in significant comminution of the original grains, as shown in Fig. 6.

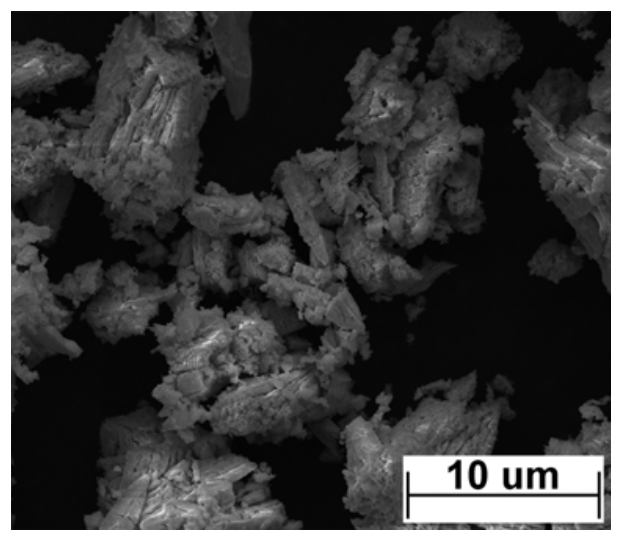

Fig. 6. Rod morphology following pressing to $130 \mathrm{MPa}$ resulting in an initial density compact of $4.0 \mathrm{~g} / \mathrm{cm}^{3}$ showing break-up of initial rod morphology.

During the pressing process the initial rods appear to be broken down into roughly two classes of particles, blocky particles of approximately $10 \mu \mathrm{m}$ diameter and smaller irregular particles that are between $2-5 \mu \mathrm{m}$. A particle configuration of this type suggests that the tips (ends) of the rods are crushed during quasi-static loading, and that the fragments of the original particles fill the voids initially left by the high aspect ratio powders. Therefore, for the dynamically loaded rods the actual configuration subjected to shock loading consists of a semi-close packed network of larger blocky particles whose interstices are partially filled with smaller irregular particles.

Thus in comparing the affect of initial morphology on the higher pressure shock compaction response for the $300 \mathrm{~nm}$ particles (56-11-56) and rods (56-11-55), one is effectively comparing the shock response in a semihomogeneous network of fine particles $(<1 \mu \mathrm{m})$ against that in a strongly bi-modal network of larger blocky particles $(\sim 10 \mu \mathrm{m})$ and smaller irregular particles $(2-5 \mu \mathrm{m})$. For these two configurations, one would expect a significant variation in localized pressures at the shock front as the material is transformed into the high pressure state. For the network of fine particles the number of contact points over which pressure is transferred is large, and consequently the pressure at each contact point is reduced. For the rods, which are composed of larger particles, the number of contact points at the shock front are far fewer, and consequently the local pressure at each contact point is higher. Therefore, one means by which to explain the observed variation in compaction response between the rods and the $300 \mathrm{~nm}$ powder is through a localized pressure approach, which is dependent largely on size. Irregularities in particle shape can also lead to a reduced coordination and a fewer number of contact points for which the shock front can propagate. Examining particle shape, it is observed in Fig. 1(a) and Fig. 6 that the coordination is more likely to be affected by irregularity in particle shape for the rods. Thus it is thought that both the larger particle size, coupled with the increased irregularity in particle shape are the dominant morphological aspect that result in the rods being more easily compacted than the $300 \mathrm{~nm}$ powder at higher pressures. 


\section{Conclusions}

In this work the compaction response of three distinct morphology $\mathrm{CeO}_{2}$ powders was investigated. In the lowstrain-rate compaction regime, quasi-static densification curves suggest that the initial stages of compaction, e.g. initial packing efficiency and rearrangement under the application of applied pressure, have the greatest influence on the final densities achievable. It was found that during the initial stages of compaction nearly all aspects of initial morphology are important, inclusive of particle size and size distribution, shape, surface roughness. At higher strain-rates, current data suggests that effects of pre-processing must be considered when analyzing compaction trends. When pre-compacted to $4.0 \mathrm{~g} / \mathrm{cm}^{3}$ minimal variation in the consolidation response for the three morphology powders was observed. For morphologies where a direct comparison in compaction response could be observed (300 nm and rods), the primary morphological traits that affect comption are found to be particle size and irregularity of shape, which influence pressure localization at the shock front.

\section{References}

1. V. Kenkre, M. Endicott, S. Glass, A. Hurd, J. Amer. Ceram. Soc. 79, (1996) 3045-3054.

2. E. Santiso, E. Müller, Molecular Phys. 100 (2002) 2461-2469.

3. R. Yang, R. Zou, A. Yu, J. Appl. Phys. 94, (2003) 3025-3034.

4. C.E. Morris, in Shock Waves in Condensed Matter 1981, AIP Conference Proceedings (AIP, New York 1982) 616-620.

5. O. Strand, D. Goosman, C. Martinez, T. Whitworth, Rev. Sci. Instrum. 77, (2006) 083108-1.

6. A. Mitchell, W. Nellis, J. Appl. Phys. 52 (1981) 33633374.

7. L. Barker, R. Hollenbach, J. Appl. Phys. 41 42084226.

8. D.A. Fredenburg, D. Dennis-Koller, D. Dattelbaum, in Shock Compression of Condensed Matter - 2011, AIP Conference Proceedings (AIP, New York) in press. 